\title{
Intraperitoneal marsupialization of a giant para-pelvic renal cyst: A case report
}

\author{
Abdul Rehman, MD; Muhammad Awais, MD; ${ }^{+}$Noor UI-Ain Baloch, MD; Hammad M. Ather, $M D^{\varsigma}$
}

*Department of Surgery, Aga Khan University Hospital, Karachi, Pakistan; †Department of Radiology, Aga Khan University Hospital, Karachi, Pakistan; §Section of Urology, Department of Surgery, Aga Khan University Hospital, Karachi, Pakistan

Cite as: Can Urol Assoc J 2014;8(11-12):e872-4. http://dx.doi.org/10.5489/cuaj.2072 Published online November 24, 2014.

\section{Abstract}

Simple renal cysts are among the most common cystic lesions of the kidney. Renal cysts most often remain asymptomatic, but rarely these cysts may become huge in size and result in significant symptoms. Giant renal cysts present a technical challenge in terms of surgical management. Although laparoscopic procedures have been performed successfully at experienced centres, intraperitoneal marsupialization via open approach may be a feasible option at less experienced centres. We report a case of a young female with a giant $(24 \times 18 \mathrm{~cm})$ renal cyst of Bosniak type-Il variety. De-roofing and intraperitoneal marsupialization of the cyst was performed. At the 1-year follow-up, she was asymptomatic, with no residual cyst.

\section{Introduction}

Simple renal cysts are among the most common cystic lesions of the kidney. ${ }^{1}$ An overall prevalence of $11.9 \%$ has been reported, with frequency increasing with age. ${ }^{2,3}$ The natural history of these cysts increases in size ${ }^{2}$ and renal dysfunction and hypertension are considered risk factors. ${ }^{4}$ Most often these occur sporadically, but they can be associated with certain diseases. Polycystic kidney disease is the most common among these disorders followed by von-Hippel Lindau syndrome and tuberous sclerosis. ${ }^{1}$

Various imaging modalities have been used to characterize renal cysts. Ultrasonography and computed tomography $(\mathrm{CT})$ are most often used and can differentiate between simple and complex cysts. ${ }^{1}$ Bosniak proposed a classification of renal cysts based on their radiographic appearances. ${ }^{5}$

Simple renal cysts often remain asymptomatic. However, they may occasionally enlarge and result in hydronephrosis, hypertension, and even polycythemia. ${ }^{6}$ Surgical treatment is indicated only if the cyst becomes symptomatic or if it is suspicious for malignancy. ${ }^{7}$ Numerous surgical approaches to manage renal cysts have been described, including open, laparoscopic ${ }^{8}$ and retroperitoneoscopic ${ }^{9}$ procedures. We report the intraperitoneal marsupialization of a giant parapelvic renal cyst - a rarely performed procedure.

\section{Case report}

A 27-year-old female presented to the outpatient department complaining of abdominal swelling for the past 4 months and abdominal pain for the past month. The swelling was more noticeable on the left side of the abdomen and increased progressively over time. Abdominal pain was localized to the left hypochondrium and left lumbar regions, aggravated by lying straight and relieved by standing up. She also reported having loose watery stools for the past 2 months, along with a sensation of epigastric discomfort and retrosternal burning. Her medical history was unremarkable.

On physical examination, her vitals were within normal range and general physical examination was only remarkable for conjunctival pallor. On abdominal examination, a lump was palpable, starting from epigastrium to just above the umbilicus and extending into the left hypochondrium and left lumbar region. The swelling was mobile with a smooth surface and firm consistency, not attached to the overlying skin or any deeper structures. The rest of the systemic examination was unremarkable.

A CT scan was performed, which showed a well-defined large para-pelvic cyst originating from the left kidney with thick enhancing septa. The cyst measured $24 \times 18 \mathrm{~cm}$ in size with significant displacement of adjacent structures (Fig. 1). The lesion was classified as a Bosniak type-Il renal cyst. Surgical treatment was advised in view of the significant symptoms and size of the cyst.

Preoperative work was unremarkable. She was approached via subcostal flank incision. The left ureter was identified and ureterotomy was done. Methylene blue was then injected, 


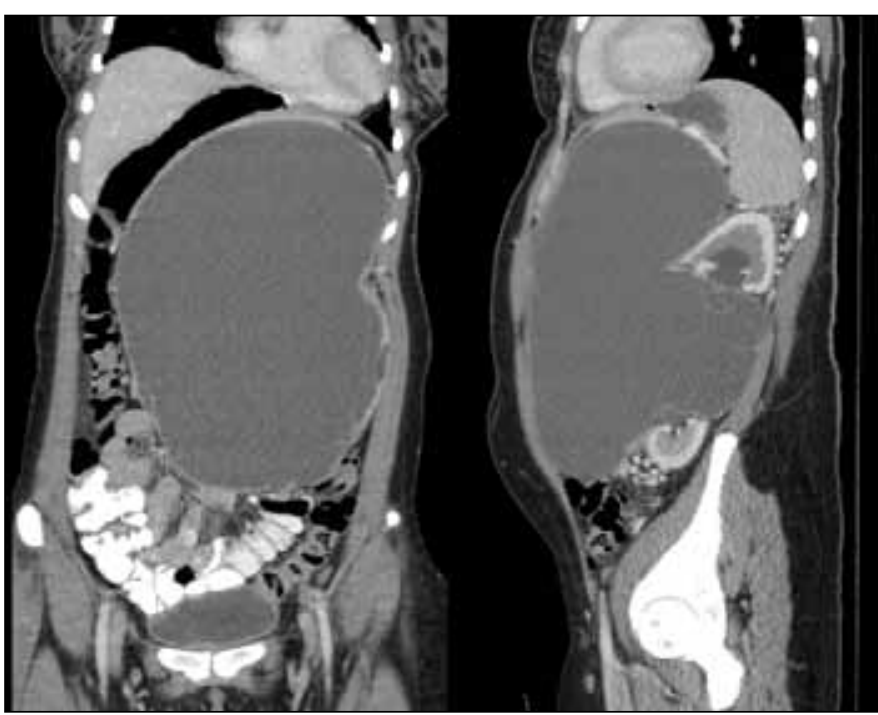

Fig. 1. Computed tomography scan of our patient. Coronal and sagittal views of computed tomography of the abdomen and pelvis demonstrate a large cyst arising from the interpolar region of left kidney. The cyst measures $24 \times 18 \mathrm{~cm}$ in craniocaudal and transverse dimensions, respectively.

which revealed no communication of the cyst with the collecting system. More than $2.5 \mathrm{~L}$ of greenish fluid was drained from the cyst and a window was created within the parietal peritoneum. Marsupialization of the cyst was done, draining the cyst cavity into the peritoneal cavity.

Postoperatively, the patient remained well and she was subsequently discharged on postoperative day 3. Culture and sensitivity of the cyst fluid did not reveal any growth of microbes. Cytology revealed mainly red blood and inflammatory cells against a proteinaceous background with no evidence of malignancy.

\section{Discussion}

Renal cysts in young people tend to grow rapidly. ${ }^{2}$ In our case, the patient was only 27 years old. Multiloculated cysts are also known to have accelerated growth. ${ }^{2}$ A CT of our patient showed multiple thick contrast-enhancing septa within the cyst, which may explain the large size of the cyst.

Giant renal cystsmeasuring more than $15 \mathrm{~cm}$ in size are extremely rare. ${ }^{6}$ The cyst in our case was $24 \mathrm{~cm}$ in craniocaudal and $18 \mathrm{~cm}$ in transverse dimensions respectively - very rare. The sheer size of the cyst was responsible for significant compression of adjacent structures, which made the cyst symptomatic.

The CT scan of our patient showed a significant shift of the bowel loops to the right of the abdomen. This possibly resulted in alteration of bowel motility and might explain the presence of loose watery stools in our patient. Moreover, compression of the stomach superiorly may have caused nausea and epigastric discomfort.
Giant renal cysts are also known to cause obstruction of the renal collecting system and subsequent hydronephrosis. ${ }^{1}$ In our patient, however, the CT revealed only mild hydronephrosis. This might be explained by the fact that the cyst in our case was para-pelvic and did not cause significant compression of the collecting system.

Hypertension, secondary to compression and segmental ischemia of the kidney with subsequent renin release, has been reported in few cases of giant renal cysts. ${ }^{3}$ Hypertension was not observed in our case. This may also be explained by the para-pelvic nature of the cyst.

Surgery has been traditionally considered the mainstay of treatment for giant renal cysts. ${ }^{7}$ Recently, percutaneous aspiration of renal cysts along with injection of sclerosing agents (such as alcohol) have been explored as a potential treatment option. ${ }^{10}$ This approach of percutaneous management can be effective, especially if the cyst is relatively small and para-pelvic in location; however, if more than $200 \mathrm{~mL}$ of the original volume persists after this procedure, then surgery becomes necessary. Retroperitoneal approach for open or laparoscopic surgical decortications is well-established. ${ }^{9}$ Although laparoscopy has been used frequently in cases of giant renal cysts, ${ }^{8}$ such procedures are only to be undertaken at centres with vast experience in laparoscopic surgery. Open surgical approach is preferred at less developed centres, where expertise with laparoscopy is not available. In our case, successful marsupialization of the giant parapelvic renal cyst was performed with no immediate or late postoperative complications. Intraperitoneal marsupialization of giant renal cyst with creation of a peritoneal window is a rarely reported procedure in the literature and this case demonstrates the feasibility of this approach.

\section{Conclusion}

Giant renal cysts are exceedingly rare and present a technical challenge for the urologist. Albeit laparoscopic approaches have been used safely at experienced centres, open surgery may also be performed. Creation of a peritoneal window and intraperitoneal marsupialization of the cyst in such cases may be a feasible option, especially at less developed centres.

Competing interests: Dr. Rehman, Dr. Awais, Dr. Baloch and Dr. Ather declare no competing financial or personal interests.

This paper has been peer-reviewed. 
Rehman et al.

\section{References}

1. Kreft B, Schild HH. Cystic renal lesions [in German]. Rofo 2003;175:892-903. http://dx.doi. org/10.1055/s-2003-40425

2. Tereda N, Ichioka K, Matsuta Y, et al. The natural history of simple renal cysts. J Urol 2002;167:21-3. http://dx.doi.org/10.1016/S0022-5347(05)65373-6

3. Altarac S. Simple renal cysts [in German]. Lijec Visen 2004;126:260-3.

4. Tereda N, Arai Y, Kinukawa N, et al. Risk factors for renal cysts. BJU Int 2004;93:1300-2. http:// dx.doi.org/10.1111/i.1464-410X.2004.04844.x

5. Israel GM, Hindman N, Bosniak MA. Evaluation of cystic renal masses: Comparison of CT and MR imaging by using the Bosniak classification system. Radiology 2004;231:365-71. http://dx.doi.org/10.1148/ radiol.2312031025

6. Pejicic T, Hadzi-Diokic J, Markovic B, et al. Resolving erythrocytosis and hypertension after open surgical extirpation of giant renal cyst measuring $30 \mathrm{~cm}$ : Case report. Ren Fail 2011;33:249-51. http://dx.doi. org/10.3109/0886022X.2011.552148
7. Agarwal MM, Hermal AK. Surgical management of renal cystic disease. Curr Urol Rep 2011;12:3-10. http://dx.doi.org/10.1007/s1 1934-010-0152-2

8. Hemal AK. Laparoscopic management of renal cystic disease. Urol Clin North Am 2001;28:115-26. http://dx.doi.org/10.1016/S0094-0143(01)80013-6

9. Lutter I, Weibl P, Daniel I, et al. Retroperitoneoscopic approach in the treatment of symptomatic renal cysts. Bratisl Lek Listy 2005;106:366-70.

10. Skolarikos A, Laguna MP, de la Rosette JJ. Conservative and radiological management of simple renal cysts: A comprehensive review. BJU Int 2012;110:170-8. http://dx.doi.org/10.1111/i.1464410X.2011.10847.x

Correspondence: Dr. Abdul Rehman, Department of Surgery, Aga Khan University Hospital, Karachi, Pakistan; jsmawais@yahoo.com 